\title{
Identification of Particle-Particle Interactions in Suspension Polymerization Reactors
}

\author{
Eric J. Hukkanen and Richard D. Braatz \\ University of Illinois at Urbana-Champaign
}

\begin{abstract}
Suspension polymerization is commonly used to produce micron-sized (10-1000 $\mu \mathrm{m})$ polymer beads, in which the final particle size distribution is an important end-use property. This paper presents an integrated approach for the modeling, simulation, and parameter estimation of particleparticle dynamics during suspension polymerization. This approach integrates in situ particle size measurement with a high resolution finite volume algorithm to estimate the kinetics of particle-particle interactions while accounting for polymerization kinetics and viscoelastic effects. The resulting simulation model is sufficiently computationally efficient to enable the numerical solution of optimal control problems based on first-principles models of suspension polymerization.
\end{abstract}

\section{INTRODUCTION}

In suspension polymerization reactions, a relatively hydrophobic monomer is dispersed as liquid droplets by a combination of vigorous stirring and suspending agents (e.g., poly(vinyl alcohol) (PVA) and hydroxyethyl cellulose (HEC)) [1]. The aqueous suspending agents are used to control coalescence during the early stages of polymerization and agglomeration during the later stages. The reaction takes place within each droplet, which has approx. $10^{8}$ free radicals [2] and therefore the polymerization is described by bulk polymerization kinetics. As the polymerization proceeds, the droplets become polymer beads.

Suspension polymerization has been investigated for decades and numerous reviews have been written on the subject [1], [3]. A major objective for suspension polymerization reactions is to control the final particle size distribution [3]. Suspension polymerization is used commercially to produce polystyrene, poly(methyl methacrylate), poly(vinyl chloride), poly(vinyl acetate), and poly(acrylonitrile). This method of polymerization is advantageous due to its easy temperature control, low dispersion viscosity, low levels of impurities within the polymer product, and low separation costs, and that the final product is in particle form [3].

In this study, experiments were conducted to accurately identify the kinetics of the particle-particle dynamics during suspension polymerization. To the best of authors' knowledge, no past papers report the direct identification of the kinetics of particle-particle interactions during suspension polymerization, due to limitations in in-situ sensors for measuring particle size distributions (PSDs) and because solving the population balance equations for the droplet/particle

Currently with Mettler-Toledo, Inc

Corresponding author. Tel.: (217)333-5073, Fax: (217)333-5052, Email: braatz@uiuc.edu. (R. D. Braatz) size distribution was considered as being too computationally expensive [4]. The parameter estimation algorithm in this study integrates two in-situ PSD sensors with a high resolution simulation algorithm, which enables the first direct identification of the kinetics of particle dynamics in suspension polymerization.

This paper develops coupled simulation models for bulk and suspension polymerization. This coupling takes into account the physical changes that the monomer droplets undergo as the reactions progress. Parameter sensitivity analysis is applied to quantify the affect of perturbations to specific parameters on the droplet/particle size distribution and corresponding moments of the distribution. This is the first investigation of such an integrated approach for the modeling, simulation, and parameter estimation of the kinetics of particle-particle interactions during suspension polymerization.

\section{Model IDENTIFICATION}

The particle size distribution (PSD) evolution is governed by

$$
\begin{array}{r}
\frac{\partial f(L, t)}{\partial t}+\frac{\partial}{\partial L}[G(L, t) f(L, t)]= \\
\int_{L}^{\infty} \nu(\lambda) b(\lambda) P(L, \lambda) f(L, t) d \lambda-b(L) f(L, t) \\
+\frac{L^{2}}{2} \int_{0}^{L} \frac{c\left(\lambda^{\prime}, \lambda\right)}{\lambda^{\prime 2}} f\left(\lambda^{\prime}, t\right) f(\lambda, t) d \lambda \\
\quad-f(L, t) \int_{0}^{\infty} c(L, \lambda) f(\lambda, t) d \lambda(1)
\end{array}
$$

which accounts for the rate of change of droplets/particles of size $L$ by breakage and coalescence, where

$$
\lambda^{\prime}=\left(L^{3}-\lambda^{3}\right)^{1 / 3} .
$$

\section{A. Droplet/Particle Growth Rate}

The particles undergo size-dependent growth according to free radical polymerization kinetics:

$$
G(L, t)=\varepsilon \frac{L_{o}}{3} \frac{d x}{d t}
$$

where $L_{o}$ is the initial droplet diameter, $x$ is the monomer conversion, and $\varepsilon=\left(\rho_{m}(T)-\rho_{p}(T)\right) / \rho_{p}(T)$ is the volume contraction factor that takes into account the density differences between the polymer and monomer. The contraction factor and monomer conversion are coupled to free radical 
bulk polymerization kinetics such that the molar balances for the batch system are

$$
\begin{gathered}
\frac{1}{V} \frac{d\left(\left[I_{2}\right] V\right)}{d t}=-k_{d}\left[I_{2}\right] \\
\frac{1}{V} \frac{d([I \cdot] V)}{d t}=-k_{i}[I \cdot][M]+2 f k_{d}\left[I_{2}\right] \\
\frac{1}{V} \frac{d([M] V)}{d t}=-k_{i}[I \cdot][M]-k_{p}[M][P]-k_{t r m}[M][P] \\
\frac{1}{V} \frac{d\left(\left[P_{1}\right] V\right)}{d t}=k_{i}[I \cdot][M]-k_{t r m}[M]\left(\left[P_{1}\right]-[P]\right) \\
-k_{p}[M]\left[P_{1}\right]-\left(k_{t c}+k_{t d}\right)\left[P_{1}\right][P] \\
\frac{1}{V} \frac{d\left(\left[P_{n}\right] V\right)}{d t}=k_{p}[M]\left(\left[P_{n-1}\right]-\left[P_{n}\right]\right)-k_{t r m}[M]\left[P_{n}\right] \\
\left.\frac{1}{\frac{d\left(\left[D_{n}\right] V\right)}{d t}=}+k_{t d}\right)\left[P_{n}\right][P] \text { for } n=2, \ldots \\
\frac{1}{2} k_{t c} \sum_{m=1}^{n-1}\left[P_{m}\right]\left[P_{n-m}\right] \\
\text { for } n=2, \ldots, \infty
\end{gathered}
$$

where $I_{2}$ is the initiator, $I$ - is the initiator radical, $M$ is the monomer, $P_{n}$ and $D_{n}$ are the live and dead polymer chains with $n$ monomer units. The parameters $k_{d}, k_{i}, k_{p}, k_{t r m}, k_{t c}$, and $k_{t d}$ are rate constants and $f$ is the initiator efficiency. The quasi-steady state approximation (QSSA) is assumed, in which the concentration of initiator radical, $I \cdot$, is constant during the entire reaction. Therefore, the molar balance for the initiator radical is

$$
\frac{1}{V} \frac{d([I \cdot] V)}{d t}=0
$$

which implies that

$$
k_{i}[I \cdot][M]=2 f k_{d}\left[I_{2}\right] .
$$

The total concentration of live polymer molecules and monomer units are respectively defined by

$$
[P]=\sum_{j=1}^{\infty}\left[P_{j}\right]
$$

and

$$
[M]=\frac{\left[M_{o}\right](1-x)}{1+\varepsilon x}
$$

where $x$ is the conversion. Throughout the reaction, the volume of the reactant/product mixture will change according to

$$
\begin{gathered}
V=V_{0}(1+\varepsilon x) \\
\frac{d V}{d t}=V_{0} \varepsilon \frac{d x}{d t} .
\end{gathered}
$$

In this work, the gel effect or "Trommsdorf effect" is accounted for using free volume correlation [5]. The void volume in a monomer/polymer solution is defined as the free volume. As conversion increases, the free volume decreases. Established methods are implemented to account for the decrease in propagation and termination due to strong diffusion limitations. The overall termination rate, $k_{t}=k_{t d}+k_{t c}=k_{t}^{o} g_{t}$, where

$$
g_{t}= \begin{cases}0.10575 e^{17.15 \nu_{f}-0.01715(T-273.15)}, & \nu_{f}>\nu_{f, c r} \\ 0.23 \times 10^{-5} e^{75 \nu_{f}}, & \nu_{f} \leq \nu_{f, c r}\end{cases}
$$

and the propagation rate $k_{p}=k_{p}^{o} g_{p}$ where

$$
g_{p}= \begin{cases}1, & \nu_{f}>\nu_{f, c r} \\ 7.1 \times 10^{-5} e^{71.53 \nu_{f}}, & \nu_{f} \leq \nu_{f, c r}\end{cases}
$$

The free volume, $\nu_{f}$, is defined by

$$
\nu_{f}=\phi_{m} \nu_{f, m}+\phi_{p} \nu_{f, p}
$$

where $\phi_{m}$ and $\phi_{p}$ are the volume fractions of monomer and polymer and the monomer $\nu_{f, m}$ and polymer $\nu_{f, p}$ free volumes are defined by

$$
\begin{aligned}
\nu_{f, m} & =0.025+0.001(T-167), \\
\nu_{f, p} & =0.025+0.00048(T-387) .
\end{aligned}
$$

The critical free volume is defined by

$$
v_{f, c r}=0.1856-2.965 \times 10^{-4}(T-273.15) .
$$

The free volume model is adapted from [5].

The viscosity of the monomer/polymer droplet increases as the monomer conversion increases. Several methods have been used to describe this phenomenon. In this study, a modified version of the free-volume theory to account for monomer/polymer viscosity [6] is applied:

$$
\mu_{d}(x, T)=\mu_{d o}(T) \exp \left[\frac{4.577 \phi_{p}}{A(T)+B_{v} \phi_{p}}\right]
$$

where $\mu_{d}(x, T)$ is the zero-shear viscosity that is dependent on conversion $x$, and temperature $T, \mu_{d o}(T)$ is the temperature-dependent viscosity of pure monomer,

$$
\phi_{p}=x /(1+\varepsilon x)
$$

is a dimensionless polymer concentration, and $A(T)$ and $B$ are model parameters used in free-volume theory [7] where

$$
\begin{gathered}
A(T)=0.15998+7.812 \times 10^{-6}(T-387.1)^{2} \\
B_{v}=0.03 .
\end{gathered}
$$

\section{B. Droplet Breakage and Coalescence}

1) Turbulence: By applying Kolmogoroff's theory of isotropic turbulence, energy-dissipating eddies in stirred tanks are independent of the main flow pattern. The rate of dissipated power is given by $\delta=N_{p} \omega^{3} D_{I}^{5} / V_{t}$ where $N_{p}$ is the power number, $D_{I}$ is the diameter of the impeller, and $V_{t}$ is the tank volume. For this study, the power number is absorbed into $\delta$, such that $\hat{\delta}=\omega^{3} D_{I}^{5} / V_{t}$. The microscale length of the turbulence is $L_{m}=\left(\nu^{3} / \delta\right)^{1 / 4}$ where $\nu=\mu / \rho$ is the kinematic viscosity of the tank contents. For a droplet 
diameter $L \geq L_{m}$, the relative velocity $u$ between two points separated by $L$ is given by [8]

$$
u(L) \propto \frac{\delta^{1 / 3} L^{1 / 3}}{1+\phi}
$$

where $\phi$ is the monomer fraction.

2) Droplet/Particle Breakage Rate: The breakage rate is described by the expression

$$
b(L)=\omega_{b}(L) e^{-\lambda_{b}(L)} .
$$

The overall breakage rate is dependent on breakage frequency, $\omega_{b}$, and efficiency, $\lambda_{b}$ [8]. The breakage frequency is described by

$$
\omega_{b}(L)=k_{b} \frac{\omega}{(1+\phi)}\left(\frac{D_{I}}{L}\right)^{2 / 3}
$$

where $k_{b}$ is the unknown breakage frequency parameter, $\omega$ is the stirring speed, $\phi$ is the monomer fraction, $D_{I}$ is the diameter of the impeller, and $L$ is the particle size.

The breakage efficiency is described using a Voigt model to account for the droplet viscosity. The breakage efficiency is the ratio of a critical energy to the turbulent kinetic energy. The turbulent kinetic energy is proportional to the particle volume, density and average relative velocity. The critical energy considers the Voigt model and contributions of elastic stress from interfacial tension and viscous stress. The breakage efficiency is

$$
\lambda_{b}=\frac{a_{b} \sigma(1+\phi)^{2}}{\rho_{d} L^{5 / 3} \hat{\delta}^{2 / 3}}+\frac{b_{b} \eta_{d}(1+\phi)}{\rho_{d} L^{4 / 3} \hat{\delta}^{1 / 3}}
$$

where $a_{b}$ is the breakage efficiency parameter associated with interfacial tension, $\sigma$ is the interfacial tension at the monomer/polymer-water-surfactant interface, and $\rho_{d}$ is the density of the droplet. The interfacial tension (Langmuirtype) is described by [9]

$$
\sigma=\sigma_{o}-K_{\sigma} \frac{K_{A} C_{P V A}}{1+K_{A} C_{P V A}}
$$

where $\sigma_{o}$ is the interfacial tension of the system (monomerwater), $K_{\sigma}$ and $K_{A}$ are constants, and $C_{P V A}$ is the surfactant concentration. The second part of (22) accounts for the viscous stress where $b_{b}$ is the breakage efficiency parameter associated with viscous stress and $\eta_{d}$ is the viscosity of the droplet. Therefore, the droplet breakage rate is

$$
b(L)=\frac{k_{b}}{(1+\phi) L^{2 / 3}} \exp \left[-\frac{a_{b} \sigma(1+\phi)^{2}}{\rho_{d} L^{5 / 3} \hat{\delta}^{2 / 3}}-\frac{b_{b} \eta_{d}(1+\phi)}{\rho_{d} L^{4 / 3} \hat{\delta}^{1 / 3}}\right]
$$

It is assumed that when a droplet ruptures, the number of daughter droplets $\nu(L)=2$ and the distribution of daughter droplets is described by

$$
P(L, \lambda)=\frac{2.4}{L^{3}} \exp \left[-4.5 \frac{\left(2 \lambda^{3}-L^{3}\right)^{2}}{L^{3}}\right] .
$$

TABLE I

FREE RADICAL POLYMERIZATION KINETIC PARAMETERS AND PHYSICAL CONSTANTS USED IN SUSPENSION POLYMERIZATION SIMULATIONS.

\begin{tabular}{llcc}
\hline Parameter & Value & Units & Reference \\
\hline$f$ & 1.0 & - & - \\
$k_{d}$ & $1.7 \times 10^{14} \exp \left(-3.0 \times 10^{4} / R T\right)\left[\mathrm{s}^{-1}\right]$ & {$[10]$} \\
$k_{t r m}$ & 1.236 & {$[\mathrm{~L} / \mathrm{mol} \cdot \mathrm{min}]$} & {$[11]$} \\
$k_{t d}^{o}$ & $1.9735 \times 10^{9}$ & {$[\mathrm{~L} / \mathrm{mol} \cdot \mathrm{min}]$} & {$[11]$} \\
$k_{t c}^{o}$ & 0.0 & {$[\mathrm{~L} / \mathrm{mol} \cdot \mathrm{min}]$} & {$[11]$} \\
$k_{p}^{o}$ & $3.354 \times 10^{4}$ & {$[\mathrm{~L} / \mathrm{mol} \cdot \mathrm{min}]$} & {$[11]$} \\
$R$ & 1.987 & {$[\mathrm{cal} / \mathrm{mol} \cdot \mathrm{K}]$} & - \\
$\rho_{m}(T)$ & $968.0-1.15(T-273.15)$ & {$[\mathrm{g} / \mathrm{L}]$} & {$[12]$} \\
$\rho_{p}(T)$ & $1.212 \times 10^{3}-0.845(T-273.15)[\mathrm{g} / \mathrm{L}]$ & {$[12]$} \\
$M_{w}$ & 100.12 & {$[\mathrm{~g} / \mathrm{mol}]$} & - \\
$\sigma_{o}$ & 0.01300 & {$[\mathrm{~N} / \mathrm{m}]$} & {$[13]$} \\
$K_{\sigma}$ & 0.01027 & {$[\mathrm{~N} / \mathrm{m}]$} & {$[13]$} \\
$K_{A}$ & 0.00847 & {$[\mathrm{~L} / \mathrm{g}]$} & {$[13]$} \\
\hline
\end{tabular}

3) Droplet/Particle Coalescence Rate: Similarly, the coalescence rate is described by

$$
c(L, \lambda)=\omega_{c}(L, \lambda) e^{-\lambda_{c}(L, \lambda)} .
$$

The overall coalescence rate of two droplets is dependent on coalescence frequency, $\omega_{c}$, and efficiency, $\lambda_{c}$ [8].

The coalescence frequency is described by

$$
\omega_{c}(L, \lambda)=k_{c} \frac{\omega D_{I}^{2 / 3}}{1+\phi}\left(L^{2}+\lambda^{2}\right) \sqrt{L^{2 / 3}+\lambda^{2 / 3}} .
$$

where $k_{c}$ is the coalescence frequency parameter, $\omega$ is the stirring speed, $D_{I}$ is the diameter of the impeller, $\phi$ is the monomer fraction, and $L$ and $\lambda$ are the diameters of the droplets. The coalescence efficiency [8] is described by

$$
\lambda_{c}(L, \lambda)=a_{c} \frac{\eta_{d} \rho_{d} \hat{\delta}}{\sigma^{2}(1+\phi)^{3}}\left(\frac{L \lambda}{L+\lambda}\right)^{4}
$$

where $a_{c}$ is the coalescence efficiency parameter, $\eta_{d}$ is the viscosity of the droplet, $\rho_{d}$ is the density of the droplet, and $\sigma$ is the interfacial tension of the monomer-water-surfactant system. Therefore, the overall coalescence rate is

$$
\begin{gathered}
c(L, \lambda)=k_{c} \frac{\omega D_{I}^{2 / 3}}{1+\phi}\left(L^{2}+\lambda^{2}\right) \sqrt{L^{2 / 3}+\lambda^{2 / 3}} \times \\
\exp \left[-a_{c} \frac{\eta_{d} \rho_{d} \hat{\delta}}{\sigma^{2}(1+\phi)^{3}}\left(\frac{L \lambda}{L+\lambda}\right)^{4}\right] .
\end{gathered}
$$

Values for kinetic parameters and physical constants are reported in Table $\mathrm{I}$.

\section{Parameter Estimation}

The objective function for parameter estimation is

$$
\min _{\theta \geq 0} \Phi(\theta)
$$

with the parameters $\theta=\left[k_{b}, a_{b}, b_{b}, k_{c}, a_{c}\right]^{T}$. The objective function equally weighs the first six moments:

$$
\Phi(\theta)=\sum_{i=1}^{N} \sum_{j=1}^{M_{i}} \sum_{k=1}^{6}\left(\frac{\mu_{k, i j}-\tilde{\mu}_{k, i j}}{\mu_{k, i j}}\right)^{2}
$$


TABLE II

EXPERIMENTAL CONDITIONS FOR ISOTHERMAL $\left(50^{\circ} \mathrm{C}\right)$ SUSPENSION POLYMERIZATION OF MMA.

\begin{tabular}{ccccccc}
\hline Exp. & $m_{M M A}(\mathrm{~g})$ & $m_{\mathrm{H}_{2} \mathrm{O}}(\mathrm{g})$ & $m_{B P O}(\mathrm{~g})$ & $\omega(\mathrm{rpm})$ & $\phi$ & $C_{P V A}(\mathrm{wt} \%)$ \\
\hline 1 & 120.0 & 280.0 & 1.20 & 450 & 0.3 & 3.0 \\
2 & 120.0 & 280.0 & 1.20 & 350 & 0.3 & 3.0 \\
3 & 120.0 & 280.0 & 1.20 & 200 & 0.3 & 3.0 \\
4 & 40.0 & 360.0 & 0.40 & 450 & 0.1 & 3.0 \\
5 & 40.0 & 360.0 & 0.40 & 350 & 0.1 & 3.0 \\
6 & 40.0 & 360.0 & 0.40 & 200 & 0.1 & 3.0 \\
7 & 120.0 & 280.0 & 1.20 & 350 & 0.3 & 1.0 \\
8 & 40.0 & 360.0 & 0.40 & 350 & 0.1 & 1.0 \\
9 & 40.0 & 360.0 & 0.40 & 200 & 0.1 & 1.0 \\
\hline
\end{tabular}

where $\mu_{k, i j}$ is the $k^{t h}$ measured moment and $\tilde{\mu}_{k, i j}$ is the $k^{t h}$ moment of the model prediction of the $j^{\text {th }}$ measurement of the $i^{t h}$ experiment, $N$ is the number of experiments, and $M_{i}$ is the number of measurements in the $i^{\text {th }}$ experiment. The $i^{\text {th }}$ moment of a droplet/particle size distribution is defined by

$$
\mu_{i}=\int_{0}^{\infty} D^{i} f(D) d D .
$$

The parameter covariance matrix $\mathbf{V}_{\theta}$ and $95 \%$ confidence intervals were computed using the standard method involving linearization of the model near the vicinity of the estimate [14]. The linear correlation matrix $\mathbf{r}$ measures the degree of linear dependence between a pair of parameters. A magnitude of 1 represents perfect correlation and magnitude of 0 represents no correlation. The coefficients of the correlation matrix [14] are given by

$$
r_{i j}=V_{\theta, i j}\left(V_{\theta, i i} V_{\theta, j j}\right)^{-1 / 2} .
$$

The diagonal entries are 1. When all off-diagonal entries have a magnitude of 0.9 and larger, the parameters are highly correlated [14].

Sequential quadratic programming was used to solve the objective function (31), where a high resolution algorithm was used to solve the PBE (1) (see [11] for details). For this specific system, the objective function (31) is many orders-of-magnitude more sensitive to the breakage parameters, indicating that it is important that these parameters be estimated accurately. The parameters associated with coalescence did not significantly affect the objective function (31) and need not be considered during parameter estimation.

\section{EXPERIMENTAL PROCEDURE}

\section{A. Materials and Instruments}

Isothermal suspension polymerization reactions were conducted in a 500-mL jacketed round-bottom flask (see Fig. 1). A single flat-blade stirrer was used to agitate the vessel. Reactants were methyl methacrylate (MMA) as dispersed phase, benzoyl peroxide (BPO) as free radical initiator, deionized water as the continuous phase, and poly(vinyl alcohol) (PVA) (88\% hydrolyzed, $\left.\bar{M}_{w} \approx 96,000\right)$ as the surfactant.

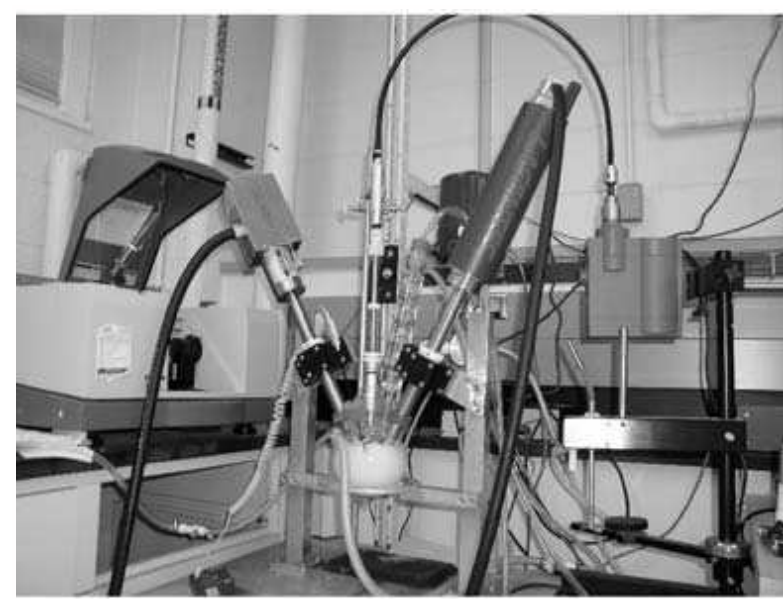

Fig. 1. Experimental set-up for suspension polymerization reactions. The in situ Lasentec FBRM (left) and Lasentec PVM (right) probes are used to measure the PSD.

The reactor was charged in the following manner and reaction conditions are reported in Table II. The controlled variables are stirring speed, $\omega$, concentration of PVA, $C_{P V A}$, and monomer fraction, $\phi=$ $m_{M M A} /\left(m_{M M A}+m_{H_{2} O}\right)$. Aqueous PVA solution was added to the jacketed round-bottom flask. The specified amounts of MMA, $m_{M M A}$, and BPO, $m_{B P O}$, were combined and added to the reactor via syringe. Upon completion of each experiment at $50^{\circ} \mathrm{C}$, the reactor was cooled to $20^{\circ} \mathrm{C}$ to quench the reaction. The reaction mixture was vacuumfiltered and dried. Details on the numerical algorithms to convert the laser backscattering data from FBRM and image data from PVM into particle size distribution measurements are described in a thesis [11].

\section{RESULTS}

All parameter estimation procedures were compiled and executed on the Linux clusters using 50 processors and a parallel version of ParticleSolver [15]. The increased number of processors, rather than executing programs in serial, showed significant increase in speed and faster turnaround on parameter estimation procedures and analysis. The final parameter estimates and confidence intervals are reported in Table III, where the covariance matrix is

$$
\mathbf{V}_{\theta}=\left[\begin{array}{ccc}
k_{b} & a_{b} & b_{b} \\
2.86 \times 10^{-13} & 4.11 \times 10^{-10} & 1.46 \times 10^{-7} \\
4.11 \times 10^{-10} & 1.37 \times 10^{-3} & -9.20 \times 10^{-3} \\
1.46 \times 10^{-7} & -9.20 \times 10^{-3} & 1.53 \times 10^{-1}
\end{array}\right]
$$

and the correlation matrix, $\mathbf{r}$, is

$$
\mathbf{r}=\left[\begin{array}{ccc}
k_{b} & a_{b} & b_{b} \\
1.000 & 0.021 & 0.699 \\
0.021 & 1.000 & -0.635 \\
0.699 & -0.635 & 1.000
\end{array}\right]
$$


TABLE III

DROPLET BREAKAGE PARAMETER ESTIMATES AND 95\% CONFIDENCE INTERVALS FOR ALL EXPERIMENTS, AS DETERMINED BY (31).

\begin{tabular}{ccc}
\hline Estimate & Value & Units \\
\hline$k_{b} \times 10^{6}$ & $8.055 \pm 1.496$ & {$\left[\mathrm{~min}^{-1}\right]$} \\
$a_{b}$ & $0.1728 \pm 0.1036$ & - \\
$b_{b}$ & $1.664 \pm 1.096$ & - \\
\hline
\end{tabular}
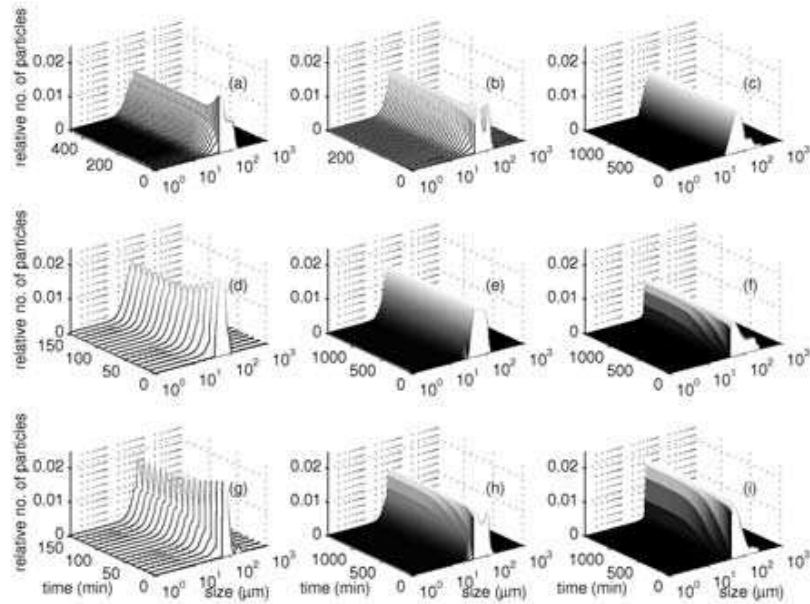

Fig. 2. High resolution finite volume simulation of full droplet/particle size distribution during suspension polymerization reactions of varying experimental conditions: (a) 1, (b) 2, (c) 3, (d) 4, (e) 5, (f) 6, (g) 7, (h) 8, and (i) 9 .

Fig. 2 reports the droplet/particle size distributions simulated for all experimental conditions (see Table II) using the final parameter estimates. Most notable in Fig. 2 is the increase in the number of smaller droplets/particles as the reaction time increases, due to breakage. Since it is difficult to visually compare the simulated experimental conditions (full distribution) to experimental results, moment ratios $\left(\mu_{1} / \mu_{0}-\mu_{6} / \mu_{0}\right)$ are used to compare experimental data and simulations in Figs. 3-5. While extensive comparisons have been made for each experiment [11], only a subset of these results are presented here due to page limitations.

Except for Experiment 7, the experiments can be interpretted in terms of three sets of three, with each set having constant experimental conditions except for the use of a different mixing speed for each experiment in the set (see Table II). The model predictions underestimated the moments for Experiments 1-2 (e.g., see Fig. 3), with Experiment 3 at low stirring speed providing the best fit to the data. The fits were best for Experiments 4-6 (e.g., see Fig. 4). The model predictions had some qualitative differences with the experimental data for Experiments 79. For example, the ratio of the lowest order experimental moments in Fig. 5 dips at 200 min which is not seen in the model predictions, so there is a bias for longer time. The rest of the experimental and simulation moment ratios are quite coincident, certainly within the stochastic variations in the data.
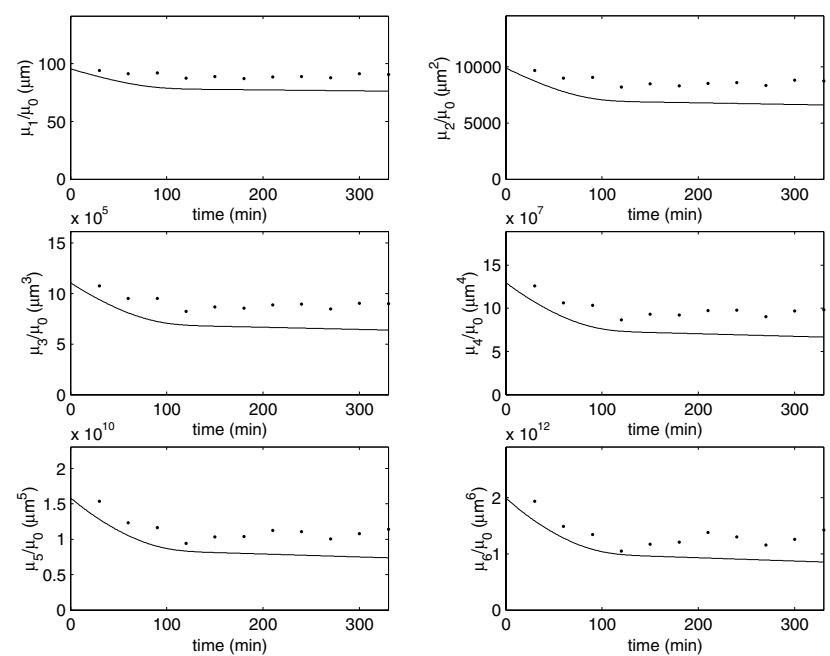

Fig. 3. Comparison of experimental droplet/particle moments $(\cdot$.$) and$ high resolution finite volume simulation of droplet/particle moments (-) for Experiment 2. The moments were calculated by applying (32) to the full droplet/particle size distribution reported in Fig. $2 b$.
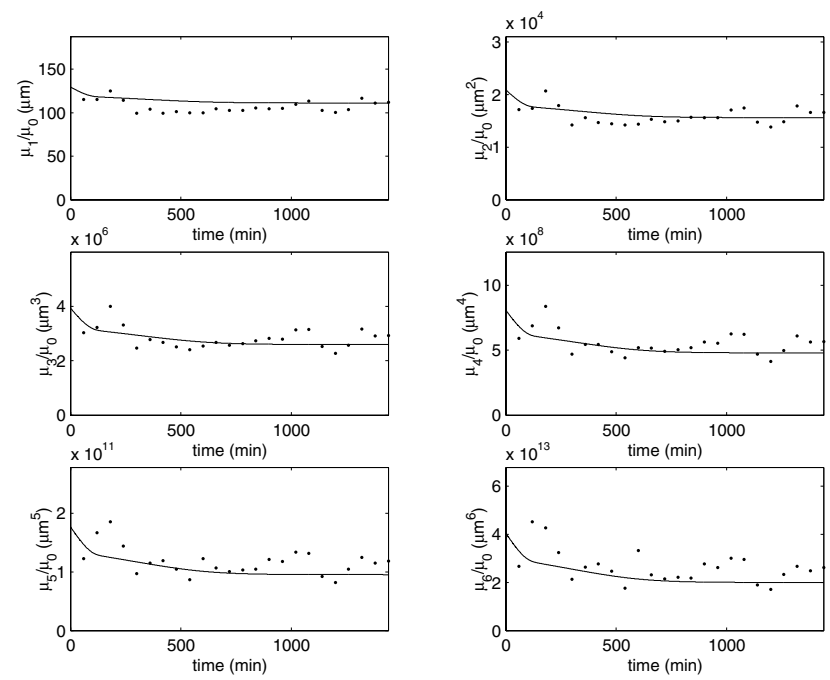

Fig. 4. Comparison of experimental droplet/particle moments $(\cdots)$ and high resolution finite volume simulation of droplet/particle moments (-) for Experiment 6 . The moments were calculated by applying (32) to the full droplet/particle size distribution reported in Fig. $2 f$.

This section presented the results of the parameter estimation procedure used to determine breakage frequency and efficiency parameters that govern particle-particle interactions during suspension polymerization. Parameter sensitivity analysis indicated that droplet breakage, and not coalescence, was the dominant mechanism in this suspension polymerization. This does not prove that coalescence does not exist, only that it is not observable. The probes used to measure droplet sizes, FBRM and PVM, were positioned near the center of the reactor (i.e., in close proximity to the stirring blade). Some theory dictates that the reactor would be better modeled as having two regions: 

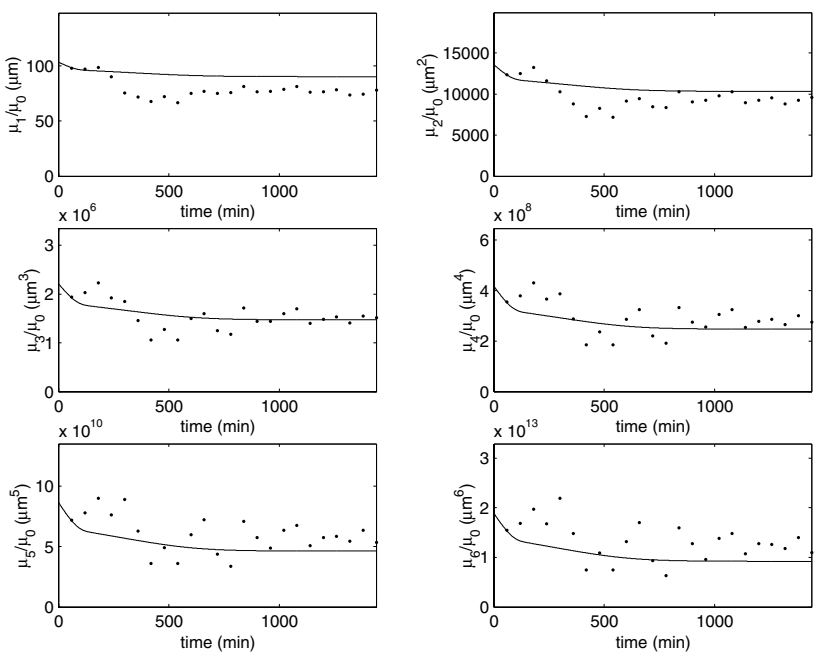

Fig. 5. Comparison of experimental droplet/particle moments $(.$.$) and$ high resolution finite volume simulation of droplet/particle moments (-) for Experiment 9. The moments were calculated by applying (32) to the full droplet/particle size distribution reported in Fig. $2 \mathrm{i}$.

the impeller region where droplet breakage is dominant and a circulation region where coalescence is dominant [1]. In this case the system would not be homogeneous, and mixing and transport between the regions should be considered. For future work it is suggested to position an FBRM and PVM probe near the wall of the reactor, to determine the suitability of the two-regions model.

\section{CONCLUSIONS}

Suspension polymerization is commonly used to produce micron-sized $(10-1000 \mu \mathrm{m})$ polymer beads. The final particle size distribution is an important end-use property. This paper presented a systematic approach to model a suspension polymerization reactor. Accurate droplet/particle size distributions were measured using in situ laser backscattering and video microscopy. A population balance equation was used to model the particle size distribution evolution during suspension polymerization and bulk polymerization kinetics were used to model the droplet growth rate and viscosity. A high resolution finite volume algorithm was used to numerically integrate the PBE on parallel computers. The efficient finite volume algorithm made a rigorous parameter estimation and sensitivity analysis procedure possible, in which it was determined that droplet breakage was dominant and that coalescence kernels did not significantly affect the final PSD. There was good agreement between the model prediction and experiments. Such first-principles model for suspension polymerization of MMA-BPO-PVA enable the formulation of optimal control problems.

\section{REFERENCES}

[1] E. Vivaldo-Lima, P. E. Wood, A. E. Hamielec, and A. Penlidis, "An updated review on suspension polymerization," Ind. Eng. Chem. Res., vol. 36, pp. 939-965, 1997.
[2] G. Kalfas and W. H. Ray, "Modeling and experimental studies of aqueous suspension polymerization processes. 1. Modeling and simulations," Ind. Eng. Chem. Res., vol. 32, pp. 1822-1830, 1993.

[3] H. G. Yuan, G. Kalfas, and W. H. Ray, "Suspension polymerization," Rev. Macromol. Chem. Phys., vol. 31, pp. 215-299, 1991.

[4] Z. Chen, W. Pauer, H.-U. Moritz, J. Prüss, and H.-J. Warnecke, "Modeling of the suspension polymerization process using a particle population balance," Chem. Eng. Technol., vol. 22, pp. 609-616, 1999.

[5] R. T. Ross and R. L. Laurence, "Gel effect and free volume in the bulk polymerization of methyl methacrylate," AIChE Symp. Ser., vol. 72, pp. 74-79, 1976.

[6] J. Alvarez, J. Alvarez, and M. Hernández, "A population balance approach for the description of particle size distribution in suspension polymerization reactors," Chem. Eng. Sci., vol. 49, pp. 99-113, 1994.

[7] W. Y. Chiu, G. M. Carratt, and D. S. Soong, "A computer model for the gel effect in free-radical polymerization," Macromolecules, vol. 16, pp. 348-357, 1983.

[8] C. A. Coulaloglou and L. L. Tavlarides, "Description of interaction processes in agitated liquid dispersions," Chem. Eng. Sci., vol. 32, pp. 1289-1297, 1977.

[9] A. W. Adamson, Physical Chemistry of Surfaces. New York: Wiley, 1976.

[10] G. Kalfas, H. Yuan, and W. H. Ray, "Modeling and experimental studies of aqueous suspension polymerization processes. 2. Experiments in batch reactors," Ind. Eng. Chem. Res., vol. 32, pp. 18311838, 1993.

[11] E. J. Hukkanen, "A systems approach to the modeling and control of molecular, microparticle, and biological distributions," Ph.D. dissertation, Univ. of Illinois at Urbana-Champaign, 2004.

[12] N. Tefera, G. Weickert, and K. R. Westerterp, "Modeling of free readical polymerization up to high conversion. I. A method for the selection of models by simultaneous parameter estimation," $J$. of Applied Polymer Science, vol. 63, pp. 1649-1661, 1997.

[13] N. Lazrak, N. L. Bolay, and A. Ricard, "Droplet stabilization in high holdup fraction suspension polymerization," Eur. Polym. J., vol. 34, pp. 1637-1647, 1998.

[14] J. V. Beck and K. J. Arnold, Parameter Estimation in Engineering and Science. New York: Wiley, 1977.

[15] R. Gunawan, I. Fusman, and R. D. Braatz, "ParticleSolver," 2003, computer software, available at http://brahms.scs.uiuc.edu. 